\title{
Investigation of facet-dependent InGaN growth for core-shell LEDs
}

\author{
Ionut Girgel ${ }^{\mathrm{a}}$, Paul R. Edwards ${ }^{\mathrm{b}}$, Emmanuel Le Boulbar ${ }^{\mathrm{a}}$, Duncan W. E. Allsopp ${ }^{\mathrm{a}}$, \\ Robert W. Martin ${ }^{\text {, }}$, Philip A. Shields*a \\ aDepartment of Electronic and Electrical Engineering, University of Bath,U.K., BA2 7AY; \\ bepartment of Physics, SUPA, University of Strathclyde, Glasgow, U.K., G4 ONG
}

\begin{abstract}
In this work we used vertically aligned GaN nanowires with well-defined crystal facets, i.e. the $\{11-20\}$ a-plane, $\{10-10\}$ m-plane, (0001) c-plane and \{1-101\} semi-polar planes, to investigate the impact of MOVPE reactor parameters on the characteristics of an InGaN layer. The morphology and optical characteristics of the InGaN layers grown of each facet were investigated by cathodoluminescence (CL) hyperspectral imaging and scanning electron microscopy (SEM). The influence of reactor parameters on growth rate and alloy fraction were determined and compared. The study revealed that pressure can have an important impact on the incorporation of $\mathrm{InN}$ on the $\{10-10\} \mathrm{m}$-plane facets. The growth performed at $750^{\circ} \mathrm{C}$ and $100 \mathrm{mbar}$ led to a homogeneous high $\mathrm{InN}$ fraction of $25 \%$ on the $\{10-10\}$ facets of the nanowires. This work suggests homogeneous good quality $\mathrm{GaN} / \mathrm{InGaN}$ core-shell structure could be grown in the near future.
\end{abstract}

Keywords: core-shell, InGaN, m-plane, non-polar, cathodoluminescence, nanowires

\section{INTRODUCTION}

Most commercial blue LEDs use InGaN quantum wells as their active region that have been grown in the [0001] direction, i.e. parallel to the c-plane. These have inherent limitations due to built-in electric fields along the [0001] direction which lead to reduced electron-hole overlap in the active quantum well region as a result of the quantumconfined Stark effect (QCSE).

Avoiding QCSE in polar materials normally requires the quantum wells (QWs) to be grown perpendicular to the polar axis; on either the non-polar $\{11-20\}$ and $\{10-10\}$ planes in wurtzite materials. Another option in the III-nitrides is to grow InGaN quantum wells on semi-polar crystallographic planes, especially possible planes at or close to an angle of $\sim 45^{\circ}$ to the polar axis ${ }^{1}$. However growth of III-nitrides on such non-polar and semi-polar planes is less mature than for the (0001) plane, with $\{10-10\}$ growth typically requiring bulk substrates to avoid high levels of stacking fault defects ${ }^{2}$.

Core-shell ${ }^{3,4,5} \mathrm{GaN} / \mathrm{InGaN}$ nanostructures are of considerable interest due to their high surface-to-volume ratio ${ }^{6}$ and low defective non-polar planes or facets, which are not subject to the detrimental QCSE. Such nanostructures can be grown with 1) a bottom-up approach using selective area epitaxy $y^{7,8,9}$ or 2) using a top-down approach ${ }^{10,11}$ in which nanowires with controlled aspect ratio are etched from a planar film before the re-growth of GaN/InGaN shell layers over the nanowires ${ }^{3,11,12}$

In contrast to planar layer growth, uniform InGaN growth on nanostructures is difficult because the three-dimensional (3D) growth mode leads to facet-dependent growth rates and indium nitride incorporation, leading to emission at multiple peak wavelengths ${ }^{3,4,12,13}$, InGaN growth on pre-etched GaN NWs has also been shown to be non-uniform for closely packed arrays ${ }^{12,14}$, indicating a likely dependence of indium nitride incorporation on nanorod height and spacing.

In this paper we report the growth of InGaN layers on etched nanowire (NW) arrays after the crystallographic facets have been recovered by an initial GaN growth step. The emission properties of InGaN layers grown under different conditions are compared in order to understand their influence on the InGaN growth on the different facets. 


\section{EXPERIMENT}

\subsection{Fabrication of template for MOVPE re-growth}

Nanowire arrays were created with a top-down approach ${ }^{10}$ from a planar GaN/AlN/Si(111) wafer. A pattern of nickel dots, obtained by using nanoimprint lithography and a lift-off process ${ }^{15}$, was used as a hard mask and a highly anisotropic $\mathrm{Cl}_{2} / \mathrm{Ar}$ plasma etch process was used to form the array of $\mathrm{GaN}$ nanowire cores. Nanoimprint lithography was used due to it being a high-resolution and low-cost method of nano-pattern generation ${ }^{15}$. The pattern consisted of a hexagonal array of $\mathrm{Ni}$ dots with $600 \mathrm{~nm}$ diameter on a pitch of $2 \mu \mathrm{m}$ and the plasma process resulted in NWs etched through the GaN and the AlN buffer layer into the $\mathrm{Si}(111)$ substrate.

Figure 1a shows a schematic of the cylindrical GaN nanowires made by this process. The GaN film was $1.5 \mu \mathrm{m}$ thick with an additional $0.5 \mu \mathrm{m}$ buffer layer of AlN used to manage the lattice strain within the GaN. Etching the NWs down into the Si substrate has the advantage that the etched $\mathrm{Si}$ surface can be converted into a $\mathrm{SiN}_{\mathrm{x}}$ selective growth mask, as seen in Figure 1a, through an in-situ nitridation step prior to the GaN facet recovery step. This leads to GaN re-growth only on the etched cylindrical GaN cores, as shown in scanning electron microscope (SEM) images in Figures $1 \mathrm{~b}$ and c.

a)

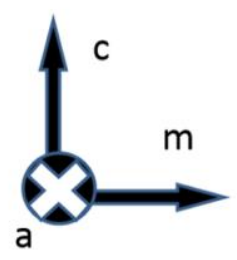

SiNx

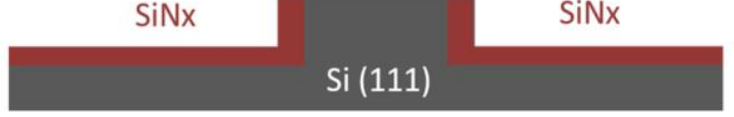

b)
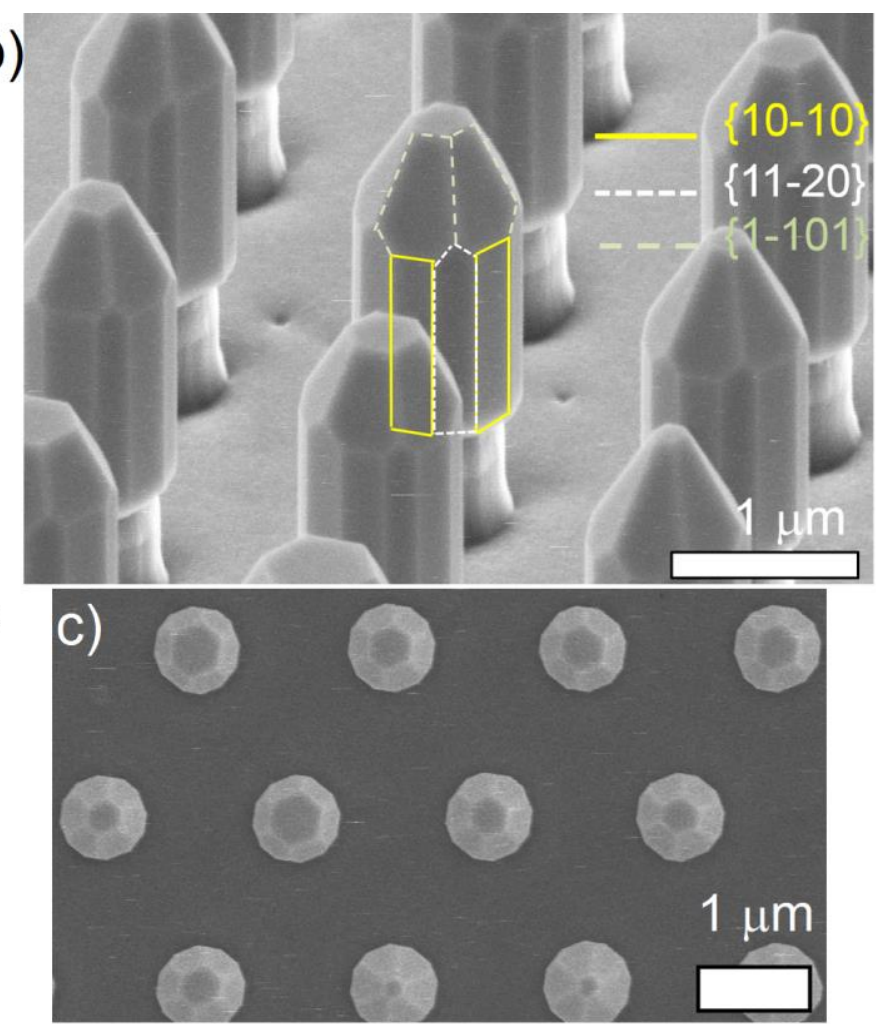

Figure 1. a) Schematic of cross-section NW after GaN re-growth on the initial etched GaN core, b) tilted secondary-electron SEM image of NWs with smooth non-polar facets and a small remaining flat c-plane, and c) plan view NW array with $2 \mu \mathrm{m}$ pitch.

\subsection{MOVPE re-growth}

After the nitridation step, performed at $1050{ }^{\circ} \mathrm{C}$ for 10 minutes and a short GaN nucleation step, a GaN facet recovery step was performed in the MOVPE reactor at $1060{ }^{\circ} \mathrm{C}$ and 50 mbar for 5 minutes in order to ensure uniform and flat nonpolar facets. These growth conditions were chosen to retain as many crystal planes as possible, in particular the $\{11-20\}$ plane as shown in Figure 1b. No GaN growth can be observed on the AlN buffer layer or on the Si between the NWs, the latter confirming the effectiveness of the $\mathrm{SiN}_{\mathrm{x}}$ selective growth mask. For the subsequent InGaN re-growth the reactor parameters determine the relative facet growth rates and the $\mathrm{InN}$ fraction incorporation on the respective facets. The TMIn and TMGa flows were kept constant across all the InGaN growths at $200 \mathrm{sccm}$ and $9 \mathrm{sccm}$ respectively. Three different InGaN growth conditions were considered with the following parameters: $750^{\circ} \mathrm{C}$ at $300 \mathrm{mbar}, 700^{\circ} \mathrm{C}$ at 
$300 \mathrm{mbar}$ and $750^{\circ} \mathrm{C}$ at $100 \mathrm{mbar}$, while all other parameters were unchanged. The long growth time was used in order to determine reliable growth rates from measurements of the thickness of the non-polar InGaN via SEM since the incorporation rate of $\mathrm{InN}$ at temperatures in the range of $700-800^{\circ} \mathrm{C}$ is low due to desorption.

Figures 2 a-c show secondary electron SEM images of the results of the three InGaN growth runs in which the various crystal facets can be identified: (0001), $\{1-101\},\{11-20\}$ and $\{10-10\}$. Each sample was grown for 30 minutes at different reactor parameters on nominally the same facetted scaffold as shown in Figure $1 \mathrm{~b}$. GaN re-growth is accomplished with selectivity between GaN/Si because of the $\mathrm{SiN}_{\mathrm{x}}$ layer, but during InGaN deposition, there was no such selectivity, as InGaN crystals can be observed in Figures $2 \mathrm{a}-\mathrm{c}$ on the substrate surface.

\section{CHARACTERIZATION OF INDIUM GALLIUM NITRIDE LAYERS}

The morphology and optical characteristics of the InGaN layers were examined by SEM and by cathodoluminescence hyperspectral imaging (CL). Because of the short growth time, the GaN NWs show a tendency to maintain a small residual (0001) plane (Figure 1b). With further GaN growth, the slower growing semi-polar $\{1-101\}$ plane would cause the NW tips to end in a pyramid shape ${ }^{16}$ and would lead the faster growing planes to extinction ${ }^{17}$. For the InGaN growths in Figures 2a-c the residual (0001) plane shows increased surface roughness ${ }^{18}$ and nano-faceting on the sub $100 \mathrm{~nm}$ range as an intermediary step towards an eventual semi-polar $\{10-11\}$ plane.
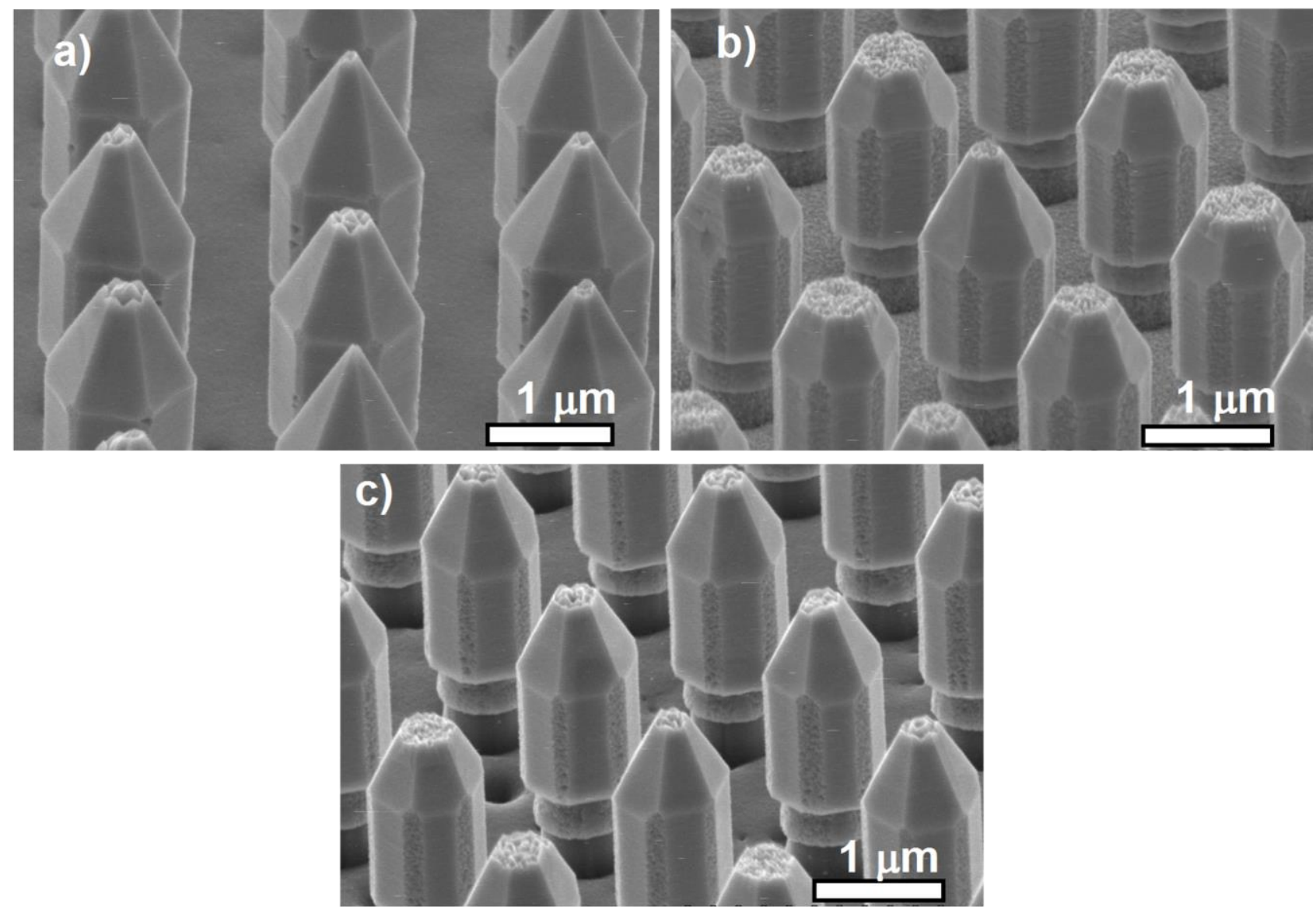

Figure 2. NWs after InGaN layer growth a) $750^{\circ} \mathrm{C} 300$ mbar shows a smooth $\{10-10\}$ plane, a rough (0001) plane and a minimized $\{11-20\}$ facet, b) $700^{\circ} \mathrm{C}$ and 300 mbar illustrates an increase in lateral size and roughness on all planes, c) $750^{\circ} \mathrm{C}$ and $100 \mathrm{mbar}$ - for the lowest growth rate the $\{10-10\}$ plane is smooth and a rough $\{11-20\}$ facet is obtained.

With most samples both the non-polar $\{11-20\}$ and $\{10-10\}$ planes are retained. The $\{11-20\}$ plane presence, which is rarely reported for nanowire structures, helps in understanding the growth rates of the relative planes and has different 
InN incorporation compared with the $\{10-10\}$ plane within the same growth. After InGaN growth on the GaN template, the $\{10-10\}$ plane surface is relatively smooth, but there is a significant increase in surface roughness for the $\{1-101\}$ plane. The high surface roughness and surface pitting ${ }^{19}$ are an indicator of that facet's faster growth rate ${ }^{20}$, while slower growing and more stable crystal planes display smooth surfaces. The changes in growth parameters had a clear impact on the crystal facets morphology, as they affect the relative growth rates related to surface energies ${ }^{21,22}$ of the various planes.

Planar secondary-electron SEM images including over $80 \mathrm{NWs}$, taken after the GaN formation stage or after the InGaN shell was added, were analyzed by image processing software, from which statistically meaningful conclusions could be extracted about the amount of growth after each of these steps. Table 1 summarizes the values of the average equivalent circular diameter of the different samples. These values represent a combination of the growth rates of the $\{10-10\}$ and $\{11-20\}$ planes. Identifying the contribution from each of the $\{10-10\}$ and $\{11-20\}$ non-polar planes to the total in a statistical manner was beyond the resolution possible with our method. For this paper it can be inferred from Figures $2 \mathrm{a}, \mathrm{b}$ that temperature has a prevailing impact on the ratio of the a- and m-plane growth rates, with the $750^{\circ} \mathrm{C}$ temperature lowering the growth rate of the $\{10-10\}$ plane in relation to the $\{11-20\}$ thus making $\{10-10\}$ a more stable facet with larger surface area compared to the $\{11-20\}$ facet. Reducing the temperature to $700^{\circ} \mathrm{C}$ had the expected effect of increasing the average non-polar facets growth rate to $8 \mathrm{~nm} / \mathrm{min}$, as the lower temperature encourages more InN integration due to the reduction of indium desorption. Reducing the pressure dramatically reduced the average growth rate to $\sim 3 \mathrm{~nm} / \mathrm{min}$. The fill factor, defined from planar SEM images as the percentage of GaN planar surface to SEM image area, is a useful indicator for comparing the proximity of neighbor NWs, as closely packed NWs led to InN fraction integration only at the tip of the $\mathrm{NWs}^{12}$.

Table 1. Comparison of growth rates corresponding to the three InGaN growths.

\begin{tabular}{|l|l|l|l|l|}
\hline Sample & $\begin{array}{c}\text { Diameter } \\
\text { (Std dev) }[\mathrm{nm}]\end{array}$ & $\begin{array}{c}\text { Diameter } \\
\text { increase }[\mathrm{nm}]\end{array}$ & $\begin{array}{c}\text { Average non-polar facets } \\
\text { growth rate [nm/min] }\end{array}$ & $\begin{array}{l}\text { Fill factor } \\
{[\%]}\end{array}$ \\
\hline GaN re-growth & $892(9)$ & & & 18.0 \\
\hline $750^{\circ} \mathrm{C} 300 \mathrm{mbar}$ & $1104(14)$ & 212 & $7.1(0.6)$ & 27.3 \\
\hline $700^{\circ} \mathrm{C} 300 \mathrm{mbar}$ & $1134(14)$ & 242 & $8.1(0.6)$ & 27.7 \\
\hline $750^{\circ} \mathrm{C} 100 \mathrm{mbar}$ & $978(14)$ & 86 & $2.9(0.6)$ & 20.4 \\
\hline
\end{tabular}

Cathodoluminescence hyperspectral imaging was used to determine the emission characteristics of the InGaN layers formed on the different facets of the NWs and to estimate their InN mole fraction using the emission energy as a function of composition ${ }^{23,24}$. Hyperspectral CL measures a full spectrum from each point on the surface scanned by the electron beam. In this work, CL spectra were collected at room temperature using a beam energy of $5 \mathrm{keV}$ and step size of $25 \mathrm{~nm}$ to create a $200 \times 200$ pixel map. Figures $3 \mathrm{a}-\mathrm{c}$ show real color images that have been determined from the chromaticity coordinates of the collected spectra and thereby illustrate the impact of the reactor conditions on the growth of the InGaN layer.

One can notice each facet has a different color due to a different InGaN composition. Corresponding spectra extracted from the same hyperspectral datasets are shown in Figure $3 \mathrm{~d}$ for the crystal planes considered. The spectra were taken on all samples from representative areas averaging $4 \times 4$ pixels from each facet. For all samples the residual (0001) plane emits in the range of $2.05-2.28 \mathrm{eV}$, although with different relative intensities. On the sample grown at $750^{\circ} \mathrm{C}$ and 300 mbar (Figure 3a), the $\{10-10\}$ plane displays emission from a smooth and uniform surface at $2.83 \mathrm{eV}$. The remaining $\{11-20\}$ plane, narrowed down due to its faster growth rate relative to $\{10-10\}$ plane, has an emission centered on $2.65 \mathrm{eV}$ and shows a further peak at higher energy, close to that of the $\{10-10\}$ peak value probably due to their proximity and consequent difficulty to achieve selective excitation by the electron beam. Interestingly the $750{ }^{\circ} \mathrm{C}$ samples show InN mole fraction incorporation on the (000-1) plane with emission at $2.30 \mathrm{eV}$, similar to that of the apices between the $\{10-10\}$ and $\{1-101\}$ semi-polar planes. The $\{1-101\}$ semi-polar facets exhibit two emission peaks, a main one centered at $2.33 \mathrm{eV}$ and a second one at $2.49 \mathrm{eV}$ at the center of the facet, the latter owing probably to migration of atom species towards the apices between $\{10-10\}$ plane and $\{1-101\}$ semi-polar facets. The true colored image showed a shift in color from the middle center to the edge of the facet. 
For the $700^{\circ} \mathrm{C} \mathrm{InGaN} \mathrm{growth} \mathrm{temperature} \mathrm{no} \mathrm{emission} \mathrm{was} \mathrm{identified} \mathrm{on} \mathrm{the} \mathrm{rough} \mathrm{a-plane,} \mathrm{possibly} \mathrm{due} \mathrm{to} \mathrm{surface}$ defects. There was a low intensity $\{10-10\}$ plane emission about $2.35 \mathrm{eV}$, possibly also due to surface defects, but the dominant emission at $2.20 \mathrm{eV}$ was from the $\{1-101\}$ semi-polar facets. Overall the decrease in temperature resulted in a red shift on all the facets, because the indium will have a lower desorption rate at this temperature.
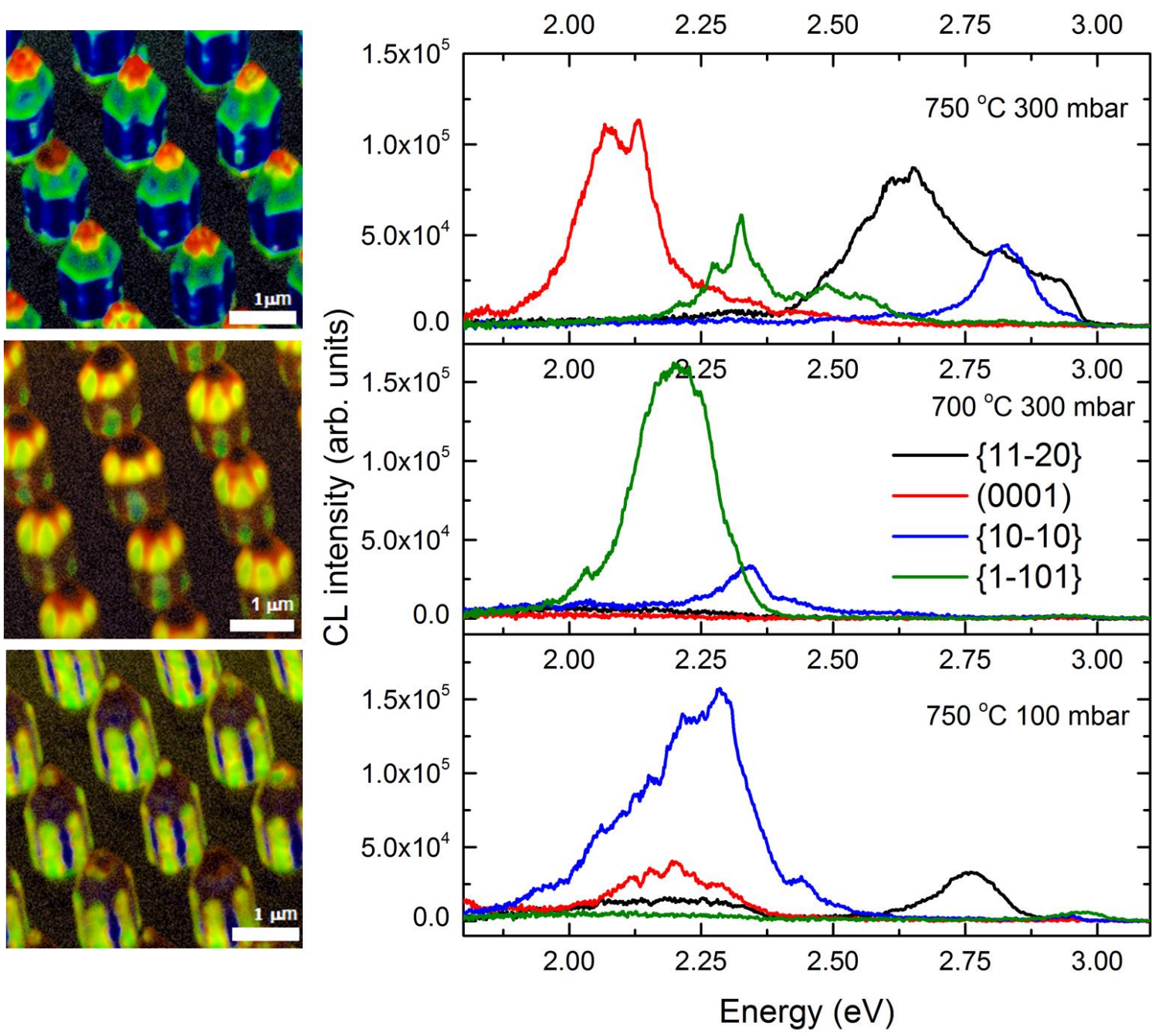

Figure 3. Real color images and corresponding CL spectra a) $750^{\circ} \mathrm{C} 300$ mbar with dissimilar emission from every facet , b) $700^{\circ} \mathrm{C} 300$ mbar sample with main emission from $\{1-101\}$ facet , c) $750^{\circ} \mathrm{C} 100$ mbar with high intensity emission from mplane facet, d) spectra collected from a representative area of $4 \mathrm{x} 4$ pixels on each facet.

The growth at $750^{\circ} \mathrm{C}$ and 100 mbar seen in Figure 3c shows substantial emission from the $\{10-10\}$ plane facets centered at $2.29 \mathrm{eV}$, and additionally a shoulder on the low energy side at approximately $2.1 \mathrm{eV}$. The $\{11-20\}$ plane shows a distinct emission at $2.75 \mathrm{eV}$ while the $\{1-101\}$ semi-polar plane is emitting at $2.97 \mathrm{eV}$ with a low intensity peak.

\section{DISCUSSION}

The results confirm that the InN fraction is strongly dependent on the growth facet. The SEM images of the initial GaN template showed smooth and complete facet recovery for all planes available as seen in Figure $1 \mathrm{~b}$. The $30 \mathrm{~min}$ InGaN growth period led to an average lateral shell thicknesses of between 43-120 nm (Table 1), perpendicular to the [0001] 
direction with growth rates from 3 to $8 \mathrm{~nm} / \mathrm{min}$, depending on reactor conditions. Each growth led to different color emission, i.e. different InN fraction was incorporated on each facet (Table 2). The emission peak energy can be used to estimate the alloy composition in separate regions of the NWs, neglecting any quantum confinement or electric field effects. The non-linearity of the InGaN band gap alloys has been reported and the indium nitride alloy composition can be determined using references ${ }^{23,24}$.

Table 2. Correlation between observed emission peaks and InN fraction.

\begin{tabular}{|l|l|l|l|l|l|l|l|l|l|}
\hline Sample & \multicolumn{3}{|c|}{$750^{\circ} \mathrm{C} 300$ mbar } & \multicolumn{3}{c|}{$700^{\circ} \mathrm{C} 300 \mathrm{mbar}$} & \multicolumn{3}{c|}{$750^{\circ} \mathrm{C} 100 \mathrm{mbar}$} \\
\hline Plane & $\{10-10\}$ & $\{11-20\}$ & $\{1-101\}$ & $\{10-10\}$ & $\{11-20\}$ & $\{1-101\}$ & $\{10-10\}$ & $\{11-20\}$ & $\{1-101\}$ \\
\hline Peak (eV) & 2.83 & 2.65 & $2.33 \& 2.49$ & 2.35 & - & 2.20 & 2.29 & 2.76 & 2.97 \\
\hline InN (\%) & 13 & 17 & $25 \& 20$ & 24 & - & 28 & 25 & 15 & 10 \\
\hline
\end{tabular}

The decrease of the growth temperature from $750^{\circ} \mathrm{C}$ to $700^{\circ} \mathrm{C}$ led to an increase of the InN fraction from $13 \%$ to $24 \%$ and $20-25 \%$ to $25-30 \%$ on both $\{10-10\}$ and $\{1-101\}$ planes. The decreased desorption rate of indium at the lower $700^{\circ} \mathrm{C}$ temperature caused the increase in InN incorporation on every facet. Also the emission from the $\{11-20\}$ and (0001) planes almost disappeared for the sample grown at $700^{\circ} \mathrm{C}$. The high $\mathrm{InN}$ incorporation or high growth rate may have induced a large number of non-radiative defects ${ }^{25}$ which decreased the intensity of the CL emission peak and broadened the full width at half maximum of the peaks. Green-to-yellow emission could be achieved using the combination of the \{10-10\} and $\{1-101\}$ semi-polar planes, which could be useful in a number of applications such as LEDs, watersplitting ${ }^{26,27}$ or solar cell technologies ${ }^{28}$.

The modification of pressure at a temperature of $750^{\circ} \mathrm{C}$ had a large impact on the growth of each facet. As can be seen in Table 2 , for $750^{\circ} \mathrm{C} 300$ mbar the average InN fraction was found to be higher in the $\{11-20\}$ plane $(17 \%)$, and $\{1-101\}$ planes (20-25\%), than in $\{10-10\}$ plane (13\%). In contrast, using a lower pressure of 100 mbar caused a change in the relative growth rates between the $\{10-10\}$ plane and $\{11-20\}$ plane, such that a greater InN mole fraction resulted on the $\{10-10\}$ plane $(25 \%)$ than on the $\{11-20\}$ plane $(15 \%)$ or the $\{1-101\}$ semi-polar facets (10\%). Such a lengthy growth time led to a wide emission peak for the $25 \%$ InN fraction, and this wide emission peak may be caused by alloy phase separation ${ }^{29,30}$, although a much richer InN phase could not be identified to validate this possibility. Alternatively the InN fraction non-uniformity could be caused by 1) a non-uniform strain distribution leading to variable incorporation rates ${ }^{31}$ or 2) the migration of atom species from the fast growing $\{10-20\}$ facets towards the $\{10-10\}$ planes.

For each growth, the $\{11-20\}$ plane surface was found to be rough. In planar films roughness is linked with high growth rates and reduced emission intensity ${ }^{20}$. Rough surface features may affect the CL intensity ${ }^{32}$ and defects cause a widening of the emission spectra ${ }^{33}$. In order to use the $\{11-20\}$ plane in a core-shell device one would need to reduce this roughness through optimization of the growth parameters so that uniform quantum wells can be created.

\section{CONCLUSION}

A GaN template of vertically aligned nanowires with (0001), $\{10-10\},\{11-20\}$ and $\{1-101\}$ facets was fabricated to study the effect of pressure and temperature on the growth characteristics of InGaN. The non-polar growth rates were determined from SEM images using image processing software. The InN mole fraction incorporation on each crystal facet was estimated by comparing the emission peak measured by CL hyperspectral imaging with previously published data $^{23,24}$. The study revealed that the reduction of pressure to 100 mbar led to a drastic change in InN fraction integration on the $\{10-10\}$ plane, increasing it from $13 \%$ to $25 \%$, while maintaining a relatively high temperature of $750^{\circ} \mathrm{C}$. This may be used to obtain green-yellow core-shell LEDs with a high InN fraction and emission from mainly the $\{10-10\}$ m-plane. In contrast there was only weak emission from the other planes. The study has shown how to control reactor parameters to deposit InN on certain facets. This understanding can guide design for core-shell LEDs. 


\section{REFERENCES}

[1] Romanov, A. E., Baker, T. J., Nakamura, S.., Speck, J. S., "Strain-induced polarization in wurtzite III-nitride semipolar layers," J. Appl. Phys. 100(2), 023522 (2006).

[2] Farrell, R. M., Young, E. C., Wu, F., DenBaars, S. P.., Speck, J. S., "Materials and growth issues for highperformance nonpolar and semipolar light-emitting devices," Semicond. Sci. Technol. 27(2), 024001 (2012).

[3] Chang, J.-R., Chang, S.-P., Li, Y.-J., Cheng, Y.-J., Sou, K.-P., Huang, J.-K., Kuo, H.-C.., Chang, C.-Y., "Fabrication and luminescent properties of core-shell InGaN/GaN multiple quantum wells on GaN nanopillars," Appl. Phys. Lett. 100(26), 261103 (2012).

[4] Yeh, T.-W., Lin, Y.-T., Stewart, L. S., Dapkus, P. D., Sarkissian, R., O’Brien, J. D., Ahn, B.., Nutt, S. R., "InGaN/GaN multiple quantum wells grown on nonpolar facets of vertical GaN nanorod arrays.," Nano Lett. 12(6), 3257-3262 (2012).

[5] Hong, Y. J., Lee, C.-H., Yoon, A., Kim, M., Seong, H.-K., Chung, H. J., Sone, C., Park, Y. J.., Yi, G.-C., "Visible-color-tunable light-emitting diodes.," Adv. Mater. 23(29), 3284-3288 (2011).

[6] Waag, A., Wang, X., Fündling, S., Ledig, J., Erenburg, M., Neumann, R., Al Suleiman, M., Merzsch, S., Wei, J., et al., "The nanorod approach: GaN NanoLEDs for solid state lighting," Phys. Status Solidi, 8, 2296-2301 (2011).

[7] Tang, T., Lin, C., Chen, Y., Shiao, W., Chang, W., Liao, C., Shen, K., Yang, C., Member, S., et al., "Nitride Nanocolumns for the Development of Light-Emitting Diode," IEEE Trans. Electron Devices 57(1), 71-78 (2010).

[8] Tomioka, K., Tanaka, T., Hara, S., Hiruma, K.., Fukui, T., "III - V Nanowires on Si Substrate : Selective-Area Growth and Device Applications,” IEEE J. Sel. Top. Quantum Electron. 17(4), 1112-1129 (2011).

[9] Liao, C.-H., Chang, W.-M., Yao, Y.-F., Chen, H.-T., Su, C.-Y., Chen, C.-Y., Hsieh, C., Chen, H.-S., Tu, C.-G., et al., "Cross-sectional sizes and emission wavelengths of regularly patterned GaN and core-shell InGaN/GaN quantum-well nanorod arrays,” J. Appl. Phys. 113(5), 054315 (2013).

[10] Shields, P., Hugues, M., Zúñiga-Pérez, J., Cooke, M., Dineen, M., Wang, W., Causa, F.., Allsopp, D., "Fabrication and properties of etched GaN nanorods," Phys. Status Solidi, 9, 631-634 (2012).

[11] Krylyuk, S., Paramanik, D., King, M., Motayed, A., Ha, J.-Y., Bonevich, J. E., Talin, A.., Davydov, A. V., "Large-area GaN n-core/p-shell arrays fabricated using top-down etching and selective epitaxial overgrowth," Appl. Phys. Lett. 101(24), 241119 (2012).

[12] Le Boulbar, E. D., Gîrgel, I., Lewins, C. J., Edwards, P. R., Martin, R. W., Šatka, A., Allsopp, D. W. E.., Shields, P. A., "Facet recovery and light emission from $\mathrm{GaN} / \mathrm{InGaN} / \mathrm{GaN}$ core-shell structures grown by metal organic vapour phase epitaxy on etched GaN nanorod arrays,” J. Appl. Phys. 114(9), 094302 (2013).

[13] Riley, J. R., Padalkar, S., Li, Q., Lu, P., Koleske, D. D., Wierer, J. J., Wang, G. T.., Lauhon, L. J., “ThreeDimensional Mapping of Quantum Wells in a GaN / InGaN," Nano Lett. 13, 4317-4325 (2013).

[14] Lewins, C. J., Le Boulbar, E. D., Lis, S. M., Edwards, P. R., Martin, R. W., Shields, P. a.., Allsopp, D. W. E., "Strong photonic crystal behavior in regular arrays of core-shell and quantum disc InGaN/GaN nanorod lightemitting diodes," J. Appl. Phys. 116(4), 044305 (2014).

[15] Shields, P. A.., Allsopp, D. W. E., "Nanoimprint lithography resist profile inversion for lift-off applications," Microelectron. Eng. 88(9), 3011-3014 (2011).

[16] Fichtenbaum, N. A., Neufeld, C. J., Schaake, C., Wu, Y., Wong, M. H., Grundmann, M., Keller, S., DenBaars, S. P., Speck, J. S., et al., "Metalorganic Chemical Vapor Deposition Regrowth of InGaN and GaN on N-polar Pillar and Stripe Nanostructures," Jpn. J. Appl. Phys. 46, 230-L233 (2007).

[17] Leung, B., Sun, Q., Yerino, C. D., Han, J.., Coltrin, M. E., "Using the kinetic Wulff plot to design and control nonpolar and semipolar GaN heteroepitaxy," Semicond. Sci. Technol. 27(2), 024005 (2012).

[18] Miao, C., Honda, Y., Yamaguchi, M., Amano, H., "Growth of InGaN/GaN multiple quantum wells on sizecontrollable nanopyramid arrays," Jpn. J. Appl. Phys. 53, 030306 (2014)

[19] Sun, Q., Yerino, C. D., Ko, T. S., Cho, Y. S., Lee, I.-H., Han, J.., Coltrin, M. E., “Understanding nonpolar GaN growth through kinetic Wulff plots,” J. Appl. Phys. 104(9), 093523 (2008).

[20] Lee, S.-N., Sakong, T., Lee, W., Paek, H., Seon, M., Lee, I.-H., Nam, O.., Park, Y., “Characterization of optical and crystal qualities in InxGa1-xN/InyGa1-yN multi-quantum wells grown by MOCVD,” J. Cryst. Growth, 250, 256-261 (2003).

[21] Northrup, J. E.., Neugebauer, J., “Theory of GaN (10-10) and (11-20) surfaces,” Phys. Rev. B 53(16), 477-480 (1996). 
[22] Neugebauer, J., “Ab initio Analysis of Surface Structure and Adatom Kinetics of Group-III Nitrides,” Phys. Status Solidi 114(1), 93-114 (2001).

[23] Davydov, V. Y., Klochikhin, A. a., Emtsev, V. V., Kurdyukov, D. a., Ivanov, S. V., Vekshin, V. a., Bechstedt, F., Furthmuller, J., Aderhold, J., et al., "Band Gap of Hexagonal InN and InGaN Alloys," Phys. Status Solidi 234(3), 787-795 (2002).

[24] O’Donnell, K. P., Fernandez-Torrente, I., Edwards, P. .., Martin, R. ., "The composition dependence of the Inx Ga1-x N bandgap,” J. Cryst. Growth 269(1), 100-105 (2004).

[25] Chiu, C. H., Kuo, S. Y., Lo, M. H., Ke, C. C., Wang, T. C., Lee, Y. T., Kuo, H. C., Lu, T. C.., Wang, S. C., "Optical properties of a-plane InGaN/GaN multiple quantum wells on r-plane sapphire substrates with different indium compositions," J. Appl. Phys. 105(6), 063105 (2009).

[26] Moses, P. G., Miao, M., Yan, Q.., Van de Walle, C. G., "Hybrid functional investigations of band gaps and band alignments for AlN, GaN, InN, and InGaN.," J. Chem. Phys. 134(8), 084703 (2011).

[27] AlOtaibi, B., Nguyen, H. P. T., Zhao, S., Kibria, M. G., Fan, S.., Mi, Z., "Highly Stable Photoelectrochemical Water Splitting and Hydrogen Generation Using a Double-Band InGaN/GaN Core/Shell Nanowire Photoanode," Nano Lett. 13, 4356-4361 (2013).

[28] Wierer, J. J., Li, Q., Koleske, D. D., Lee, S. R.., Wang, G. T., "III-nitride core-shell nanowire arrayed solar cells.," Nanotechnology 23(19), 194007 (2012).

[29] Stringfellow, G. B., "Microstructures produced during the epitaxial growth of InGaN alloys," J. Cryst. Growth 312(6), 735-749 (2010).

[30] Tessarek, C., Figge, S., Aschenbrenner, T., Bley, S., Rosenauer, A., Seyfried, M., Kalden, J., Sebald, K., Gutowski, J., et al., "Strong phase separation of strained Inx Ga1-x N layers due to spinodal and binodal decomposition: Formation of stable quantum dots," Phys. Rev. B 83(11), 115316 (2011).

[31] Li, Q.., Wang, G. T., "Strain influenced indium composition distribution in GaN/InGaN core-shell nanowires," Appl. Phys. Lett. 97(18), 181107 (2010).

[32] Martin, R. W., Edwards, P. R., O’Donnell, K. P., Dawson, M. D., Jeon, C.-W., Liu, C., Rice, G. R.., Watson, I. M., "Cathodoluminescence spectral mapping of III-nitride structures," Phys. Status Solidi 201(4), 665-672 (2004).

[33] Bertram, F., Srini, S., Liu, R., Geng, L., Ponce, F. A.., Riemann, T., "Spatial variation of luminescence of InGaN alloys measured by highly-spatially-resolved scanning cathodoluminescence,” Mater. Sci. Eng. B 93, 19-23 (2002). 